\title{
El desarrollo de competencias investigativas de los docentes en formación en el contexto de la práctica pedagógica
}

\author{
Development of Research Skills in Pre-Service Teachers \\ in the Context of the Teaching Practice
}

O desenvolvimento de competências de pesquisa dos professores em formação no contexto da prática pedagógica

\author{
Ximena Paola Buendía-Arias ${ }^{1}$ \\ Lilian Cecilia Zambrano-Castillo² \\ Edgar Alirio Insuasty ${ }^{3}$
}

\section{Resumen}

Este artículo da cuenta de los resultados de un estudio que tuvo como propósito determinar hasta qué punto los siete programas académicos de la Facultad de Educación fomentan el desarrollo de competencias investigativas en el contexto de la práctica profesional pedagógica. La recolección de la información se hizo con base en un enfoque cualitativo-descriptivo por medio de encuestas, cuestionarios, entrevistas y análisis de documentos. La técnica utilizada para analizar la información recolectada a través de estos instrumentos fue el análisis de contenido. Los principales hallazgos son la ausencia de un desarrollo balanceado de las competencias investigativas que fueron exploradas en este estudio, así como la falta de articulación entre la práctica pedagógica y la investigación. Adicionalmente, en este estudio se resalta la reconceptualización de la práctica pedagógica como un espacio para la reflexión y la investigación.

\section{Palabras clave}

competencias investigativas; facultad de educación; práctica pedagógica

\section{Abstract}

This paper presents the results of a study that aimed to determine to what point the seven academic programs of the School of Education encourage the development of research skills in the context of the professional teaching practice. The information was gathered based on a qualitative-descriptive approach through surveys, questionnaires, interviews and document analysis. The technique used to analyze the information collected through these instruments was content analysis. The main findings were the absence of a balanced development of the research skills explored in this study, as well as the lack of articulation between the teaching practice and research. The study also highlights the reconceptualization of the teaching practice as a space for reflection and research.

\section{Keywords}

research skills; school of education; teaching practice

1 Magister en Enseñanza del Inglés, Docente de planta del Programa de Licenciatura en Lenguas Extranjeras, Universidad de Sucre, Sincelejo, Colombia, ximena.buendia@unisucre.edu.co

2 Magister en Didáctica del Inglés, Profesor asociado del Programa de Licenciatura en Enseñanza del Inglés, Universidad Surcolombiana, Neiva, Colombia, licezam@usco.edu.co

3 Doctor en Educación con énfasis en Andragogía, Profesor asociado del Programa de Licenciatura en Enseñanza del Inglés, Universidad Surcolombiana, Neiva, Colombia, edalin@usco.edu.co 


\section{Resumo}

Este artigo apresenta os resultados de um estudo cujo objetivo foi determinar até que ponto os sete programas acadêmicos da Faculdade de Educação fomentam o desenvolvimento de competências de pesquisa no contexto da prática profissional pedagógica. A coleta da informação foi realizada com base em uma abordagem qualitativa-descritiva, por meio de enquetes, questionários, entrevistas e análises de documentos. A técnica utilizada para analisar a informação coletada foi a análise de conteúdo. As principais descobertas são a ausência de um desenvolvimento balanceado das competências investigativas que foram exploradas neste estudo, assim como a falta de articulação entre a prática pedagógica e a pesquisa. Adicionalmente, neste estudo assinala-se a reconceitualização da prática pedagógica como um espaço para a reflexão e a pesquisa.

\section{Palavras chave}

competências de pesquisa; faculdade de educação; prática pedagógica

Artículo recibido el 22 de septiembre de 2016 y aprobado el 28 de julio de 2017

\section{Introducción}

Al establecerse la Constitución de 1991 en nuestro país se replanteó el concepto de educación en los diferentes ámbitos que cubre. Entre otros aspectos encontramos cuáles deben ser sus fines y el nuevo papel que los actores del proceso educativo deben asumir. A partir de estos planteamientos, el Ministerio de Educación Nacional (MEN) a través de la Ley General de 1994, establece entre las finalidades de la formación de educadores "Fortalecer la investigación en el campo pedagógico y en el saber específico" (artículo 109). Esto hace evidente, como lo afirman Muñoz, Quintero y Munévar (2001) la necesidad del desarrollo de "competencias para que los educadores construyan en sus prácticas pedagógicas cotidianas un ambiente investigativo, que se preocupen por la innovación educativa y por su propia autoformación como profesionales" (p. 26). El MEN (2014), consciente de la necesidad de seguir mejorando la calidad de la educación en nuestro país, continúa formulando lineamientos para las licenciaturas, los cuales contemplan, entre otros aspectos

[...] la imperante necesidad de vincular y ajustar la investigación científica que se produce en educación a nuestras características contextuales, y formular y desarrollar proyectos que consideren las actuales y futuras prioridades de mejorar la calidad de la educación, de sus actores, de sus prácticas y del aprendizaje de sus estudiantes [...] se espera que la investigación se oriente al análisis de las prácticas, su mejoramiento y la incorporación y validación de innovaciones didácticas que aporten a los aprendizajes de los estudiantes. (p. 9).
Atendiendo a estos requerimientos, la Facultad de Educación de una universidad pública en Colombia promueve desde su misión la formación de educadores con sólidos conocimientos pedagógicos y disciplinares, con actitud crítica e investigativa, porque se reconoce que es a través de la formación investigativa como se puede alcanzar en los futuros docentes la profundización de sus conocimientos, así como una mayor autonomía intelectual y espiritual para problematizar su realidad educativa y buscar su mejoramiento.

De la misma manera los siete programas de pregrado de esta facultad propenden también por afianzar este tipo de formación a través de la experiencia de la práctica profesional docente, tal y como se puede apreciar en los objetivos del Reglamento de Práctica (Consejo de la Facultad de Educación de la Universidad Surcolombiana, 2013) en donde se establece, entre otros aspectos, que el estudiante practicante:

Desarrolle su espíritu investigativo, acorde con las tendencias actuales en educación, de tal manera que produzca cambios fundamentales, haciendo énfasis en el desarrollo de la creatividad, laboriosidad y la sensibilidad; [...] aplique elementos técnicos, investigativos conducentes a innovar procesos pedagógicos; $[. .$.$] genere procesos de$ transformación de la realidad educativa de la comunidad; [...] de soluciones específicas a los problemas identificados durante el desarrollo de la Práctica Educativa en el aula y/o en el entorno social en el que se desempeña .

De esta manera, se reconoce la importancia que tiene el escenario de la práctica como un espacio 
para empoderar a los futuros docentes, a partir de la promoción de experiencias investigativas, como transformadores de nuestra realidad educativa.

Para determinar si estas expectativas se cumplían, en el programa de Licenciatura en Inglés se desarrollaron tres estudios (Zambrano e Insuasty, 2008, 2010, 2011), orientados a establecer si la práctica pedagógica estaba siendo aprovechada como un espacio que le permitiera al estudiante reflexionar, autoevaluarse y construir su propia identidad profesional a partir de una retroalimentación efectiva por parte de sus asesores. Con base en algunos vacíos encontrados se propusieron dos intervenciones: la primera, orientada a mejorar el uso del diario y el blog como herramientas de reflexión; la segunda, a optimizar el proceso de retroalimentación de tal manera que el asesor se convirtiera en un promotor de espacios, en donde el diálogo con el estudiante practicante estuviera enfocado a fomentar una autocrítica constructiva, en la búsqueda de soluciones a la problemática que surge en el día a día de su quehacer educativo.

En un diagnóstico inicial, con base en la consulta de las percepciones de los coordinadores, asesores y practicantes de los siete programas académicos de la Facultad de Educación sobre el cumplimiento de los objetivos del Reglamento de Práctica, se logró establecer que aún se tienen dificultades para alcanzar los objetivos investigativos propuestos por esta facultad en el contexto de la práctica docente. Particularmente, un bajo pero significativo porcentaje de asesores (31\%), practicantes (19\%) y coordinadores $(14 \%)$ concibe el objetivo que tiene que ver con la "construcción y desarrollo de saberes y competencias investigativas en los procesos de enseñanza y aprendizaje" (p. 3) como una meta que requiere mayor trabajo si se espera alcanzarla en su totalidad. Otro objetivo, que se refiere a la "elaboración, ejecución y evaluación de proyectos de investigación en el aula, en comunidades y en otras instancias donde se realicen prácticas para intervenir acertadamente en ellas" (p. 3) tampoco se ha cumplido en su totalidad, según la percepción del $50 \%$ de asesores, el $30 \%$ de practicantes y el $14 \%$ de coordinadores. El cumplimiento de otros objetivos afines concernientes al incremento del espíritu inves- tigativo y a la divulgación del conocimiento científico tampoco tuvo una valoración satisfactoria.

A partir de estos resultados de carácter general, y con el propósito de valorar el desempeño investigativo de los profesores en formación durante la práctica pedagógica, la presente investigación estuvo orientada, en primer lugar, a determinar las percepciones de los actores de la práctica pedagógica (coordinadores de práctica, asesores y practicantes) sobre el desarrollo de competencias investigativas de los practicantes y, posteriormente, a caracterizar las experiencias investigativas que promueven los programas de la Facultad de Educación en la práctica pedagógica. Los resultados de este estudio pueden contribuir significativamente a optimizar los procesos de formación investigativa de nuestros estudiantes de licenciatura, a partir de la identificación de algunos vacíos que rodean la experiencia de la práctica pedagógica.

\section{Fundamentación teórica}

Puesto que este artículo se centra en el desempeño investigativo de los docentes en formación durante el desarrollo de su práctica pedagógica, en esta sección se describen brevemente los siguientes ejes temáticos: enseñanza reflexiva, investigación educativa y competencias investigativas.

\section{Enseñanza reflexiva}

La enseñanza reflexiva puede ser conceptualizada como un enfoque pedagógico que, según Pennington (citado por Farrell, 2003), busca mejorar los procesos y resultados dentro del aula de clase, como también contribuir a la formación de docentes y aprendices motivados y seguros de sí mismos. Este enfoque tiene como piedra angular el concepto de "pensamiento reflexivo" propuesto por Dewey, que corresponde "al examen activo, persistente y cuidadoso de toda creencia o supuesta forma de conocimiento a la luz de los fundamentos que la sostienen y las conclusiones a las que tiende" (1909, p. 9). Este pedagogo argumenta que el pensamiento reflexivo involucra dos subprocesos: uno es un estado de perplejidad, vacilación o duda; el otro es el 
acto de búsqueda o de investigación para encontrar algún material que esclarezca o disipe la perplejidad.

Hay tres formas de reflexión que cumplen estas características. Schön (citado por Corcoran y Leahy, 2003) habla de dos maneras diferentes en las que se puede dar la reflexión: en la acción y sobre la acción. La primera consiste en reaccionar ante una determinada situación de enseñanza-aprendizaje en el mismo momento en que ocurre. La segunda tiene lugar después de acontecida la acción y se trata de una retrospección de la clase, lo cual sirve de base para documentar, sistematizar y reflexionar las experiencias del aula. A los dos tipos de reflexión propuestos por Schön, Kilon y Todnew (citados por Farrell, 2003) añaden un tercer tipo denominado "reflexión para la acción". Esta última es una forma de consolidar las dos anteriores; es decir, mientras se examinan nuestras acciones pasadas y presentes, se genera un conocimiento para nuestras futuras acciones, a través de procesos de investigación-acción.

Uno de los contextos propicios para empoderar a los docentes en formación como profesionales reflexivos es la práctica pedagógica. Justamente Insuasty y Zambrano (2011), citando a Freeman, establecen que durante la experiencia de la práctica docente se espera que el docente en formación desarrolle la capacidad de tomar sus propias decisiones informadas acerca del proceso de enseñanza y evaluar el impacto de esas decisiones tanto en su propia formación como en el aprendizaje de sus estudiantes.

En igual sentido, Chacón (2006) reafirma la importancia de la práctica cuando establece que

[...] las prácticas constituyen espacios para la formación porque permiten interacción con las instituciones educativas y posibilitan la vinculación teórico práctica para conformar una cultura de la investigación, reflexión, transformación y sus implicaciones en la formación de ciudadanos comprometidos con el mundo dinámico de hoy. (p. 206).

Por su parte, Mercado (2003) nos invita a pensar en cómo y desde dónde abrimos espacios para que maestros y estudiantes reflexionen alrededor de lo que sucede en las prácticas pedagógicas y cómo estas pueden contribuir a los procesos de formación de los futuros maestros.

En el mismo orden de ideas, Cid, Domínguez y Raposo sugieren que

[...] entre los objetivos más importantes que se deben desarrollar en el período de la práctica docente están aquellos que le permitan al estudiante adquirir hábitos de investigación y reflexión con el fin de impulsarlo a que construya su conocimiento profesional, y así a partir de la valoración crítica de sus vivencias pueda identificar problemáticas y tomar decisiones para resolverlas. (Citados por Pérez, Benarroch, Jiménez, Smith, y Rojas, 2006, p. 35).

Finalmente, se destaca aquí la propuesta del ciclo reflexivo de Bartlett (1994) como una manera de trascender las tecnicidades de la enseñanza y pensar en mejorar nuestras acciones, preguntándonos no solo cómo abordar el proceso, sino también el qué y el porqué de aquello que sucede en nuestro contexto educativo. Para este propósito, él propone un ciclo compuesto por cinco componentes básicos que no son lineales ni secuenciales:

- Trazar el mapa. ¿Qué hago como docente? Responder este interrogante implica la observación y recolección de evidencias en el salón de clase a través del uso de diarios personales, registro de aprendizaje, portafolios y/o diarios.

- Dar significado al mapa. ¿Cuál es el significado de mi enseñanza? ¿Cuál era mi intención? Es esencialmente buscar el significado detrás de los datos recogidos. Esta fase informativa proporciona al docente una comprensión sobre la diferencia entre la enseñanza rutinaria y la acción concienzuda de enseñanza.

- Rebatir. ¿Cómo llegué a esa forma de enseñanza? ¿Cómo fue posible que surgiera mi actual concepción sobre la enseñanza? Esta fase implica refutar nuestras ideas y las estructuras que las soportan. Para este fin, los docentes pueden compartir de cierta manera sus concepciones y razones pedagógicas con sus colegas.

- Evaluar. ¿Cómo podría enseñar en una forma diferente? Esto tiene que ver con la búsqueda de 
distintas alternativas de acción que sean consistentes con nuestra "nueva" manera de entender la enseñanza; es decir, con el surgimiento de planes o estrategias orientadas a mejorar la calidad del proceso de enseñanza-aprendizaje.

- Actuar. ¿Qué y cómo debería enseñar ahora? Esto tiene que ver con la implementación de aquellos planes emergentes de enseñanza. Freire (citado por Bartlett, 1994), establece que "reflexión sin acción es palabrería, y acción sin reflexión es activismo" (p. 213).

\section{Investigación educativa}

Desde mucho tiempo atrás la necesidad de hacer investigación educativa ha sido no solo mencionada por muchos autores, sino que también se ha hecho mayor con el surgimiento de nuevos enfoques y paradigmas en el campo educativo. Muñoz, Quintero y Munévar (2001) afirman que la investigación se ha hecho prioritaria en el siglo XXI como medio para generar conocimientos. De esta manera, la investigación en educación se ha concebido como un camino viable para los entes educativos que buscan mejorar la labor docente y por ende el proceso de enseñanza-aprendizaje.

Indudablemente, la investigación educativa se ha convertido en un reto para las instituciones universitarias que buscan formar docentes investigadores, puesto que esta es la única herramienta que puede ayudar al maestro a entender su quehacer pedagógico y en consecuencia mejorarlo a través del acto reflexivo y planes de acción. Travers (citado por Ary, Jacobs y Sorensen, 2010), plantea que la investigación educativa permite adquirir información útil y confiable sobre el proceso educativo. Asimismo, Restrepo (s. f.) la define como aquella que se encuentra en lo pedagógico y busca el mejoramiento de la educación con relación al currículo, los métodos de enseñanza y otros factores tales como medios y materiales, organización y clima de la clase, y procesos de interacción o comunicación.

Muñoz, Quintero y Munévar (2001) reconocen la importancia de la investigación educativa al resaltar la urgencia de que el "profesorado genere conoci- mientos a partir de su propia práctica, a cambio de ser un usuario pasivo del conocimiento generado por otros" (p. 23). Esto es fundamental porque permite que el maestro desarrolle habilidades críticas frente a su saber y práctica pedagógica, sin tener que someterse a lo que otros proponen. Así lo justifica Calvo (2002), quien plantea que el aula es el espacio donde el docente encuentra problemas de manera singular y donde debe experimentar estrategias de intervención que solo pueden desarrollarse a través de propuestas de investigación y que surgen a partir de la reflexión sobre la práctica pedagógica.

Los docentes investigadores disponen de varios enfoques y opciones metodológicas para desarrollar una investigación educativa que contribuya al estudio, el análisis, la comprensión y el mejoramiento de las realidades focalizadas. Entre estos podemos resaltar los siguientes: investigación descriptiva, etnografía, investigación acción, investigación acción participativa, investigación histórica, investigación evaluativa, investigación ex post facto e investigación experimental.

Finalmente, cabe señalar que la investigación sobre fenómenos relacionados con la educación puede realizarse desde el punto de vista tanto cuantitativo como cualitativo (Ramírez, 2009). El desarrollo de las competencias investigativas por parte de los docentes desde cualquiera de estos dos paradigmas les permitirá darle una mayor unidad de sentido a los procesos de investigación educativa.

\section{Competencias investigativas}

Es a través de la investigación como los docentes en formación pueden abordar la experiencia de la práctica docente como una oportunidad para adentrarse en su autoconocimiento y para buscar sistemáticamente soluciones a los problemas que se presenten en el entorno educativo en el que están inmersos. Este propósito conlleva necesariamente el desarrollo de competencias investigativas por parte de los practicantes que les permita cumplir los siguientes propósitos:

- Comprender el significado, la importancia y las implicaciones de la investigación educativa en la práctica pedagógica del educador. 
- Observar, preguntar, registrar, interpretar, analizar, describir contextos y escribir textos acerca de situaciones problemáticas propias de los ambientes escolares.

- Proponer soluciones a los problemas detectados, utilizando los conceptos y los métodos de investigación, sean éstos explicativos, interpretativos o críticos.

- Argumentar sobre las relaciones que se establecen dentro de la cultura escolar y las alternativas que se dan a los problemas investigativos detectados.

- Perfeccionar las prácticas escriturales, redactando notas de campo sintetizando datos, textos y sistematizando informes de investigación acerca de un problema identificado en el aula o en la escuela. (Muñoz, Quintero y Munévar, 2001, pp. 15-16).

Se espera que los docentes en formación desarrollen los siguientes tipos de competencias investigativas básicas:

- Competencias para preguntar. Castillo (2011) señala que buscan esencialmente desarrollar la habilidad para formular interrogantes tanto en la lógica del descubrimiento (investigación cualitativa) como en la lógica de la verificación (investigación cuantitativa). En su intento por aproximarse a la realidad, el investigador cualitativo puede acudir a la utilización de preguntas etnográficas, que pueden ser descriptivas, estructurales, de contraste, hipotéticas, de repetición, de ejemplos, de experiencias, amistosas o de lenguaje émico.

- Competencias observacionales. Según Bartlett (1994), este componente es fundamental como punto de partida para que el docente, a través de sus registros, empiece a comprender y cuestionar lo que está sucediendo en sus clases. Por su parte, Castillo (2011) sugiere que constituyen la habilidad con la que el investigador es capaz de agudizar la observación para que sus percepciones sean selectivas y para que decida qué registrar.

- Competencias reflexivas. Ramírez y Bravo (citados por Ollarves y Salguero, 2009), plantean que una de las maneras que determina el abordaje del conocimiento es a través de la reflexión sobre la propia práctica profesional, asumiendo cada situación profesional como aprendizaje y como espacio propicio para la investigación. Villarini (2014) señala que el pensamiento reflexivo está orientado a la solución de problemas y la toma de decisiones eficaces y efectivas. Por su parte, García y Veleros (2012) conciben la reflexión como una práctica renovadora autoformativa que trata de la capacidad del docente para evaluar su propia acción al determinar en forma crítica avances, limitaciones y ausencias en el desarrollo de su función educativa.

- Competencias propositivas. Luque, Quintero y Villalobos (2012) las definen, junto con las competencias interpretativas y argumentativas, como competencias productivas observables que tienen un valor estratégico en la formación universitaria. Afirman que con estas competencias el investigador propone soluciones a los problemas detectados, utilizando los conceptos y los métodos de investigación, sean estos explicativos o críticos.

- Competencias tecnológicas. Montoya (2006) sugiere que las competencias investigativas tecnológicas tienen que ver con los desempeños y comportamientos relacionados con conocimientos de carácter técnico, procesos tecnológicos y funciones productivas Específicas. Como también lo manifiesta Castillo (2011), estas competencias se relacionan con la capacidad del investigador de seleccionar y manejar técnicas de recolección de datos, como también la utilización de software para el análisis de datos y la exposición de resultados.

- Competencias interpersonales. Estas, según Aular de Durán, Marcano y Moronta (2009) se conciben como la capacidad para relacionarse armónicamente entre los sujetos implicados, con un alto grado de motivación e incentivación y siempre utilizando métodos democrático-participativos cuando de liderazgo se trate. En este sentido, Ollarves y Salguero (2009) proponen que todas las actividades y decisiones necesi- 
tan la aprobación, inclusión, participación y coordinación de los miembros involucrados para generar nuevos productos, eventos o servicios confiables, ya que han sido sometidos a diversos juicios.

- Competencias cognitivas. Según lo plantea el sitio web Edukanda (s. f.), las competencias investigativas cognitivas tienen que ver con la capacidad para comprender, conocer, analizar, comparar y evaluar teorías, tendencias y metodologías generales relacionadas con el trabajo y sus características, que ayuden a los maestros en formación a desarrollar las destrezas pertinentes y afrontar así los problemas específicos que forman las peculiaridades del puesto de trabajo. Como también lo sostiene Castillo (2011), estas competencias tienen que ver con procesos cognoscitivos e intelectuales que llevan a la construcción de habilidades de pensamiento y a diversas alternativas investigativas. Es decir, observar, descubrir, consultar, interpretar y desarrollar conocimientos.

- Competencias procedimentales. Según Castillo (2011), estas competencias se refieren a la destreza del investigador en el manejo de las técnicas que se emplean durante el desarrollo de la investigación. Son el conjunto de habilidades necesarias para realizar, detectar, demostrar y poner en acción las funciones y actividades inherentes a la labor investigativa de manera precisa y eficaz. A través de estas competencias se busca que el investigador tenga la capacidad de llevar un orden secuencial en los pasos o etapas con que cuenta el proceso investigativo: el diseño, la experimentación, si es necesaria, la comprobación y por último la sistematización de los resultados obtenidos.

- Competencias analíticas. Es el conjunto de competencias que Ramírez y Bravo (citados por Ollarves y Salguero, 2009) definen como la habilidad para comprender en profundidad, dar sentido a los datos cualitativos y elaborar categorías de significado por medio de la información recolectada. Al mismo tiempo incluye la identificación de elementos del dominio cul- tural y la determinación del foco etnográfico de la investigación. Además, Hurtado (citado por Ollarves y Salguero, 2009) establece que estas competencias representan la capacidad para jerarquizar problemas detectados de acuerdo a los resultados obtenidos en el diagnóstico de necesidades registradas.

- Competencias comunicativas. Las competencias comunicativas, según Balvo (2010), son aquellas en las que el investigador demuestra su habilidad para generar y difundir conocimientos a partir de su investigación, bien sea en forma oral o escrita con un orden lógico y propiedad de los conocimientos a difundir. Ollarves y Salguero (2009) definen estas competencias como la labor emprendida por el investigador para intercambiar experiencias y a raíz de esto generar la solución de problemas específicos dentro del entorno educativo.

Con el desarrollo de todas estas competencias, los docentes en formación pueden potenciar su capacidad de respuesta frente a las exigencias conceptuales y procedimentales que impone la realización de investigaciones en el ámbito de la educación.

\section{Metodología}

Este estudio se realizó en el marco del paradigma cualitativo, pues buscó interpretar y comprender el fomento del espíritu investigativo en la práctica pedagógica a través de las experiencias de siete programas de una Facultad de Educación de una universidad colombiana. Patton (citado por Hernández, Fernández y Baptista, 2006) menciona que "los datos cualitativos son descripciones detalladas de situaciones, eventos, personas, interacciones, conductas observadas y sus manifestaciones". Los datos recolectados desde este modelo inductivo permitieron analizar el fenómeno en cuestión y determinar la manera en la que se está articulando la investigación con la práctica pedagógica.

El diseño metodológico corresponde a un estudio descriptivo que busca establecer la existencia de un fenómeno a través de su descripción explícita (Seliger y Shohamy, 1989). Hernández, Fernández 
Universidad Pedagógica Nacional

Facultad de Humanidades

y Baptista (2006) indican que este tipo de investigaciones se fundamentan más en un proceso inductivo que implica "explorar, describir y luego generar perspectivas teóricas". Señalan también que la recolección de datos consiste en obtener las perspectivas y los puntos de vista de los participantes, como sus emociones, experiencias, significados y otros aspectos subjetivos. En términos generales, según Seliger y Shohamy (1989), los estudios descriptivos sugieren el procedimiento que se muestra en la figura 1.

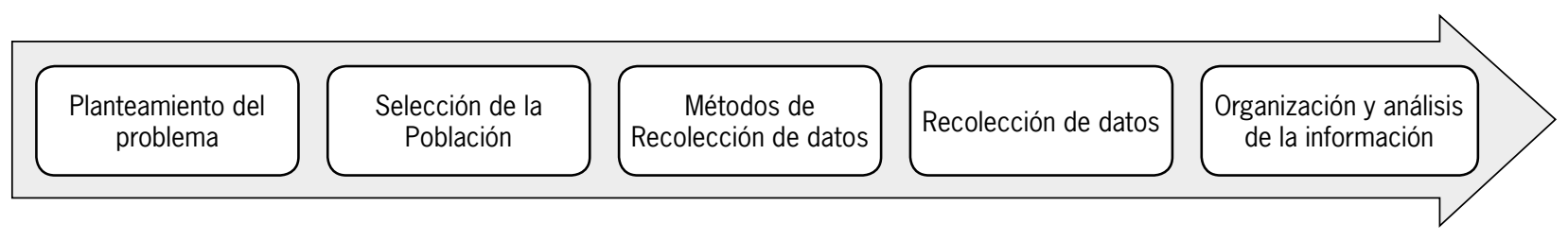

Figura 1. Etapas del proceso investigativo

\section{Participantes}

Este estudio se realizó en una universidad pública ubicada en el sur del país. Los participantes fueron los coordinadores de práctica, los practicantes y los asesores de práctica de los siete programas académicos adscritos a la Facultad de Educación de la institución: licenciaturas en Inglés, Matemáticas, Ciencias Naturales, Lengua Castellana, Educación Artística y Cultural, Educación Física y Pedagogía Infantil.

\section{Métodos de recolección de información}

Con el propósito de alcanzar los objetivos específicos, se utilizaron los siguientes instrumentos para la recolección de la información:

- Entrevista. De acuerdo con Kvale (2008) el propósito de la entrevista en la investigación cualitativa es "obtener descripciones del mundo de vida del entrevistado respecto a la interpretación de los significados de los fenómenos descriptivos". Por tanto, se aplicó una entrevista semiestructurada a los coordinadores de práctica, la cual permitió determinar sus percepciones sobre el desarrollo de competencias investigativas de los docentes en formación en el escenario de la práctica pedagógica.

- Encuestas. Se diseñaron encuestas dirigidas a los coordinadores, asesores y practicantes, que tuvieron como propósito determinar las percepciones de los encuestados con respecto al grado de cumplimiento de los objetivos de la práctica pedagógica propuestos por la Facultad de Educación . Es importante resaltar que la encuesta cualitativa busca analizar la diversidad de algún tema de interés dentro de una determinada población. Jansen (2010) señala que este tipo de encuesta no busca contar el número de personas con la misma característica, sino establecer las variaciones significativas dentro de una misma comunidad.

- Cuestionarios. Este instrumento se aplicó con el objetivo de definir el grado en el que las experiencias investigativas realizadas en la práctica pedagógica contribuyen a la consolidación de las competencias investigativas de los estudiantes. Se diseñaron dos cuestionarios diferentes: uno dirigido a practicantes y otro a asesores y coordinadores de la práctica. Rodriguez, Hoffmann y Mackedanz (2011) definen el cuestionario como una "técnica de recogida de datos que puede prestar un importante servicio en la investigación cualitativa, puesto que parte de los esquemas de referencia teóricos y permite la exploración de ideas y creencias generales sobre algún aspecto de la realidad".

- Análisis de documentos. El análisis documental es "un procedimiento sistemático usado para la revisión o evaluación de material impreso o en medio digital" (Bowen, 2009). Corbin y Strauss (2008, citados en Bowen, 2009), explican que este tipo de análisis requiere que la información se examine e interprete con el fin 
de obtener significados, adquirir mayor comprensión sobre un fenómeno y desarrollar un conocimiento empírico. Para efectos de este proyecto, los documentos analizados fueron los trabajos de investigación realizados por los practicantes de los diferentes programas de la facultad, lo cual permitió caracterizar las experiencias investigativas que promueven los respectivos programas en el contexto de la práctica docente.

\section{Análisis y validación de datos}

El análisis de contenido fue la técnica que se utilizó para analizar la información recolectada a través de los diferentes instrumentos propuestos. Porta y Silva (2003) aseveran que hoy en día este método se configura como una técnica objetiva, sistemática, cualitativa y cuantitativa que trabaja con materiales representativos, marcada por la exhaustividad y con posibilidades de generalización. El análisis de contenido se puede usar para analizar datos recolectados a través de encuestas, entrevistas, registros de observación, cuestionarios, etc. La información se analiza y se identifican categorías que posteriormente permitirán clasificar los datos. La validación de datos se realizó triangulando la información obtenida a través de las encuestas, los cuestionarios, las entrevistas y los análisis de documentos. "La triangulación ofrece la alternativa de poder visualizar un problema desde diferentes ángulos y de esta manera aumentar la validez y consistencia de los hallazgos" (Gómez y Benavides, 2005).

\section{Análisis y discusión de resultados}

Para caracterizar los proyectos de investigación de los 7 programas de la Facultad se consultó mediante entrevista a los coordinadores de la práctica, y mediante cuestionario a los asesores $y$ estudiantes acerca del tipo de proyecto que desarrollaban en la práctica pedagógica. Además, se analizaron 24 muestras de trabajos proporcionados únicamente por 4 de los 7 programas de la Facultad de Educación. El tipo de proyecto que realizan los practicantes en cada programa tiene una denomi- nación diferente: proyecto de innovación, artículo pedagógico y estudio de caso.

La caracterización de estos trabajos se hizo de acuerdo al concepto de competencia planteado por Jurado (2003), Muñoz (2001) y Aular de Duran, Marcano y Moronta (2009). Jurado (2003) asocia este término con la formación de sujetos críticos; por su parte, Borjas señala que las competencias que un docente desarrolle deben permitirle reflexionar continuamente sobre su quehacer diario, con el fin de mejorar los procesos educativos a partir de la solución de problemas que sean relevantes y pertinentes para la comunidad educativa. Finalmente, es importante retomar lo que establecen $\mathrm{Muñoz}$ (2001), Aular de Durán, Marcano y Moronta (2009) acerca de las competencias investigativas. Estos autores señalan que el desarrollo de estas debe permitir al educador observar, interpretar, preguntar, argumentar, analizar, proponer alternativas y registrar esas experiencias pedagógicas con el fin de desarrollar otras formas de comprensión de su desempeño y el de sus aprendices.

Por otra parte se tendrá en cuenta la clasificación hecha por algunos autores, como Castillo (2011), Ollarves y Salguero (2009) y Luque, Quintero y Villalobos (2012). Esta clasificación responde a las siguientes competencias investigativas: competencias observacionales y para formular preguntas, reflexivas, propositivas, tecnológicas, procedimentales, analíticas y escriturales. Las competencias cognitivas e interpersonales no se analizaron porque no se pudieron evidenciar a través de los trabajos estudiados. De acuerdo a los resultados obtenidos, el desarrollo de las competencias investigativas durante la práctica docente se podría clasificar como se propone en la tabla 1.

Tabla 1. Clasificación del fomento de competencias investigativas en la práctica docente
7 (Más fomentada)

6

5
Propositivas

Reflexivas

Procedimental 


\begin{tabular}{|c|c|}
\hline 4 & Observacionales \\
\hline 3 & Comunicativas \\
\hline 2 & Tecnológicas \\
\hline 1 (Menos fomentada) & Formular preguntas \\
\hline
\end{tabular}

\section{Competencias observacionales}

Según Bartlett (1994), la acción de observar es fundamental como punto de partida para que el docente, a través de sus registros, empiece a comprender y cuestionar lo que está sucediendo en sus clases. Según la percepción de asesores (93\%) y practicantes ( $82 \%$ ) el cumplimiento de esta acción es altamente satisfactorio. Sin embargo, algunos coordinadores expresaron que aun cuando se utilizan algunos instrumentos de observación, no se promueve la reflexión a partir de la información recopilada. Al respecto, uno de los coordinadores de práctica señala:

... lo que yo creo que nos hace falta un poco tratar, ahora que lo nombra, es el aspecto de la reflexión, si bien en algún momento tratamos de que los estudiantes llevasen un cuaderno o un diario de campo en el cual pudieran consignar algunas de sus experiencias más significativas, en eso digamos que podemos hacer mucho más, creo que es tal vez el elemento que hemos hecho menos hincapié (Coordinador del PI).

Al contrastar la opinión de los participantes con los reportes de investigación de los estudiantes, se determinó que aunque no se menciona directamente cómo se dio el proceso de observación, se puede inferir que los estudiantes, al inicio o durante su práctica, recurren a esta experiencia como punto de referencia para identificar una problemática específica del contexto educativo. Esto se puede evidenciar en algunas referencias como las siguientes: “... en la práctica pedagógica se logró establecer que en algunas de las instituciones educativas del Departamento del Huila existen dificultades de aprendizaje". Sin embargo, en algunos documentos sí se mencionan explícitamente: "A través de la observación, establecí un sinnúmero de necesidades en los niños del hogar infantil, [...] es por ello que ahora deseo realizar un estudio específico sobre el caso de alteración en los parámetros del desarrollo de..." (reporte 3 del PPI).

En cuanto a la forma como se sistematiza la información que se recoge en el periodo de observación, son muy reducidas las evidencias encontradas en los trabajos; solo algunos programas mencionan el uso de diarios o fichas valorativas. De todas maneras, como podemos observar, en estos cuatro programas sí se ofrecen espacios para desarrollar esta competencia. Sin embargo, notamos la necesidad de que todos los programas promuevan algún tipo de instrumento para sistematizar este proceso de observación a fin de que el estudiante, como lo establece Castillo (2011), perfeccione su capacidad de escoger selectivamente la información que desea registrar.

\section{Competencia para formular preguntas}

Castillo (2011) señala que al desarrollar esta competencia, el estudiante debe estar en capacidad de formular interrogantes enmarcados en la lógica del descubrimiento (investigación cualitativa) o de la verificación (investigación cuantitativa). En la mayoría de los trabajos analizados no se encontraron claramente definidas las preguntas de investigación, por lo tanto no se pudo determinar si los estudiantes plantean preguntas específicas, que se puedan responder desde la investigación, que sean relevantes y muy claras. En otros estudios se establece información como aparece en la siguiente referencia: “... lo que se busca con el proyecto es dar respuesta a una pregunta de investigación" (reporte 4 del PI); sin embargo, esta no se plantea en el reporte escrito. Solo en un documento se notó mayor claridad en la formulación de la siguiente pregunta: “¿Cómo favorece el juego del bingo periódico a la enseñanza-aprendizaje de la tabla periódica en estudiantes de grado décimo de la Institución Educativa...?" (reporte 1 del PB). Esto indica que la formulación de preguntas de investigación es un área que aún requiere atención por parte de los diferentes programas y que se debe reforzar en los cursos de investigación previos a la práctica docente. Muñoz, Quintero y Munévar (2001) afirman que para un desarrollo pleno de las competencias investigativas el practicante debe ser 
capaz de establecer preguntas acerca de situaciones problemáticas propias de los ambientes escolares.

\section{Competencias reflexivas}

Con el desarrollo de esta competencia se espera que el practicante haga una evaluación crítica de su experiencia, a partir de la cual identifica una situación problemática y mediante un seguimiento determina su impacto. Esta práctica debe asumirse como una situación profesional de aprendizaje que recrea los espacios propicios para la investigación (Ramírez y Bravo en Ollarves y Salguero, 2009). Al consultar a los participantes acerca de si se promueve el desarrollo de esta competencia como herramienta esencial para el mejoramiento de la labor docente del estudiante, asesores (100\%) y practicantes (99\%) expresaron que su cumplimiento es significativo. Solo un $1 \%$ de practicantes calificó la realización de esta acción como poco significativa. La mayoría de los coordinadores, por su parte, también afirmaron que los programas promueven el desarrollo de estas competencias no solo desde el escenario de la práctica, sino también desde los cursos que le anteceden, por ejemplo las didácticas, como se evidencia a continuación:

... tenemos los momentos en que los estudiantes se encuentran con su asesor, que es cuando hacen la atención de sus programadores, entonces hay momentos para reflexionar en torno a cómo me estoy desempeñando, ¿Qué está pasando con esa labor que estoy desarrollando allá?, ¿Cuáles han sido mis compromisos?... (Coordinadora del PLC ).

Según lo planteado por García y Veleros (2012), la reflexión se puede conceptualizar como una práctica renovadora autoformativa que trata de la capacidad del docente para autoevaluar su acción al determinar en forma crítica avances, limitaciones y ausencias en el desarrollo de su función educativa. Aquí es donde cobran importancia las orientaciones que los practicantes puedan recibir de sus asesores. En este sentido, uno de los coordinadores de práctica expresó que la reflexión y la autoevaluación no están fortalecidas dentro de su programa porque depende mucho del asesor que se den o no. El coordinador manifiesta que esta falencia se ha desarrollado porque la mayoría de asesores son catedráticos que no tienen el tiempo o la formación académica e investigativa para ayudar al practicante a formarse como docente reflexivo.

Si bien la mayoría de los participantes aseveraron que los estudiantes son alentados a asumir una postura crítica a lo largo de su experiencia en la práctica docente, los registros de la información de todos los reportes investigativos no permiten visualizar un proceso de reflexión claro, ni un seguimiento e impacto de los respectivos proyectos. Aunque el practicante no concluye de qué manera la reflexión enriqueció su formación profesional, en la mayoría de los casos se infiere que a partir de una reflexión, buscó dar solución a un problema específico que parecía afectar el aprendizaje de los discentes:

El estudio de caso fue realizado con el niño $\mathrm{x}$ de 5 años de edad el cual se trabajó sobre las "falencias en la atención y el comportamiento" que el niño venia presentando en el transcurrir del año dentro y fuera del salón de clases, convirtiéndose en una prioridad ya que se estaba afectando su desarrollo y el aprendizaje tanto de él como el de sus compañeros. (Coordinadora del PPI ).

Los resultados que surgieron relacionados con esta competencia nos muestran que sí existe la preocupación en la mayoría de los programas por formar futuros docentes equipados con ciertas herramientas que desarrollen un pensamiento reflexivo. Sin embargo, aún hay aspectos por considerar, como la falta de formación académica e investigativa de los asesores de práctica y la inexistencia de estudios en la facultad que determinen el impacto de los proyectos que desarrollan nuestros egresados en el contexto real de la práctica como fruto del proceso reflexivo e investigativo. Esto corresponde a la necesidad planteada por Jurado (2003) de que el docente desarrolle competencias que a partir de la reflexión le permitan mejorar los procesos educativos y solucionar problemas que sean relevantes y pertinentes para la comunidad educativa en general. 


\section{Competencias propositivas}

En este proceso de formación investigativa hay varias etapas que se espera que el docente practicante implemente como parte del ciclo reflexivo establecido por Bartlett (1994). No es suficiente con detenernos a pensar y registrar lo que estamos haciendo como docentes y tratar de buscar las razones que subyacen a estas acciones, e identificar algunas inconsistencias; lo que puede resultar más significativo para nuestros aprendices es buscar cómo optimizar el proceso de enseñanzaaprendizaje a través de propuestas o estrategias que puedan dar solución a esas dificultades encontradas. Según el $100 \%$ de asesores y practicantes, el estudiante practicante sí logra proponer y realizar acciones a partir de su quehacer pedagógico e investigativo, que contribuyen a generar cambios dentro o fuera del aula de clase. De igual manera, la mayoría de los coordinadores coincidieron con los demás participantes al afirmar que esta labor propositiva que se espera del practicante sí se está logrando. La coordinadora de práctica del PPI manifestó que

... lo que se pretende desde la práctica es que de alguna manera los practicantes sistematicen lo que hacen como futuros profesionales de la enseñanza de idiomas, que pongan eso por escrito, que identifiquen unas problemáticas y que propongan soluciones a esas problemáticas dentro del salón de clase.

Al contrastar estas percepciones con los reportes investigativos analizados, se determinó que en la mayoría de estas propuestas se plantean alternativas para tratar de mejorar las situaciones que están afectando de alguna manera el proceso de aprendizaje de los educandos. Como se muestra en el siguiente ejemplo: "Presento a continuación un plan de acción a ejecutarse con la niña $\mathrm{x}$ durante la práctica de intervención [...] para estimular el área del lenguaje según los parámetros establecidos a cumplir en dicha edad" (reporte 2 del PLC). Sin embargo, también se evidenció que en algunos documentos no se explica claramente en qué consiste la estrategia de intervención que se implementó.
Finalmente, estos hallazgos nos permiten inferir que los programas promueven el desarrollo de la competencia propositiva e intentan que a partir de esta, sus practicantes propendan por el cambio y el mejoramiento dentro o fuera del aula de clase con el respectivo acompañamiento de los asesores. Esto corrobora lo planteado por Calvo (2002) y Chacón (2006) quienes afirman que el aula es el espacio donde el docente debe experimentar estrategias de intervención orientadas a la transformación de los procesos de formación académica y ciudadana.

\section{Competencias tecnológicas}

Castillo (2011) señala que los desempeños que debe demostrar un estudiante al haber desarrollado esta competencia son esencialmente selección y manejo de técnicas de recolección de información, utilización de software para análisis de datos y presentación de resultados. De acuerdo a la opinión de la mayoría de los participantes (coordinadores, $90 \%$; asesores, $52 \%$ y practicantes, $83 \%$ ), quienes respondieron mediante entrevista o cuestionario, si esta competencia se desarrolla en el contexto de la práctica, y si está articulada al desarrollo de un proyecto investigativo, consideran que efectivamente sí se logra llevar a cabo esta acción. Sin embargo, al analizar la mayoría de los trabajos de los cuatro programas, no se encuentran evidencias claras del uso de recursos tecnológicos con los propósitos expuestos por Castillo. En uno de los programas solo se evidencia el uso de Excel para el diseño de gráficas; únicamente en un programa se ve reflejada una mayor práctica de estas competencias. Los autores de uno de los proyectos mencionan el uso de herramientas tecnológicas para el análisis pretest y postest, y en otro, para la sistematización de los datos obtenidos a través de cuestionarios emplearon el software Atlas.ti 7.0.

Cabe señalar también que uno de los coordinadores de práctica (PI) menciona una plataforma virtual en la cual se mantiene en contacto con los practicantes y en la que, según él, se puede compartir material bibliográfico, ideas innovadoras de actividades para realizar en clase y artículos de interés que tengan que ver con la docencia. 
No obstante, estos resultados nos permiten concluir que aún queda mucho por hacer en torno al desarrollo de esta competencia. Aun cuando los estudiantes generalmente tienen habilidades para el uso de la tecnología, no se está aprovechando este conocimiento ni se está incorporando esta herramienta en los procesos investigativos.

\section{Competencia procedimental}

Castillo (2011) define las denominadas competencias procedimentales en el sentido de constituir el conjunto de habilidades necesarias para realizar, detectar, demostrar y poner en acción las funciones y actividades permanentes, precisas, eficaces $y$ eficientes para llevar a cabo la labor investigativa. Establece además que el desarrollo de un proyecto de investigación conlleva un orden secuencial que se demuestra a través del diseño, la experimentación, si es necesaria, la comprobación y finalmente la organización del reporte escrito, estructurado a partir de ciertas etapas que le permiten al investigador sistematizar todo el proceso. Al preguntar a los participantes de este estudio acerca del cumplimiento de las diferentes etapas que conlleva el desarrollo de un proyecto investigativo, más del $75 \%$ de ellos consideran que los proyectos realizados por los practicantes sí cumplen con estos requerimientos. Sin embargo, una vez analizados los documentos mediante los cuales se presentan los reportes de los proyectos de los cuatro programas (artículo, pósters, diapositivas y reflexión escrita), se observa que solo en dos de ellos se registra la mayoría de las etapas requeridas: identificación del problema, justificación, marco teórico, metodología, resultados y conclusiones; algunos presentan una clara descripción de los resultados, especialmente en uno de los programas. No obstante, otros informes escritos se quedan cortos en el reporte del diagnóstico, el cual no se fundamenta claramente; algunos objetivos son muy generales, hay ciertos problemas en la descripción de la metodología y en el diseño de los instrumentos, y no se citan referencias bibliográficas. Esto nos permite evidenciar que aunque las licenciaturas pueden desarrollar investigación educativa desde perspectivas diferentes -cualitativa o cuantitativa como lo plantea Ramírez (2009)-, hace falta una unificación de criterios en los diferentes programas de la Facultad de Educación sobre los requerimientos para la entrega del informe escrito resultado de un proyecto de investigación, así el tipo de investigación sea diferente y se trate de un estudio a pequeña escala.

\section{Competencias comunicativas}

En el contexto de la práctica pedagógica, se espera que el estudiante practicante demuestre la habilidad "para generar y difundir conocimientos a partir de su investigación bien sea en forma oral o escrita con un orden lógico y propiedad de los conocimientos a difundir" (Balvo, 2010). A esto, Ollarves y Salguero (2009) añaden que un propósito fundamental del quehacer investigativo es intercambiar experiencias y así generar la solución de problemas específicos del entorno educativo. A este respecto, más del $70 \%$ de los participantes considera que el nivel de cumplimiento de esta competencia es significativo. Al verificar si estas percepciones se ven reflejadas en los reportes de investigación presentados por los practicantes, se pudo evidenciar que no hay unanimidad de criterios en los cuatro programas en cuanto a lo que un reporte investigativo requiere.

En primer lugar, en los cuatro programas en donde se analizaron los productos investigativos se evidenciaron diferencias en la extensión del reporte escrito: mientras unos tienen 3 páginas, otros tienen 16 y 18. Algunos reportes se presentan a manera de artículos, otros se socializan a través de pósters o presentaciones en PowerPoint. También se encontró que no todos los informes registran los mismos pasos. Algunos contienen presentación, introducción, resumen, informe de resultados y experiencias, recolección de datos, aplicación, conclusión, anexos y bibliografía; sin embargo, otros se quedan cortos en aspectos como referencias bibliográficas o diseño metodológico. Un tercer factor que surge en la revisión de estos documentos es la redacción; mientras hay algunos trabajos que se presentan dentro de los lineamientos requeridos, otros reportes evidencian una pobre redacción. Por lo tanto se puede determinar que esta competencia debería ser fortalecida. 
En cuanto a la socialización de resultados a través de presentaciones o ponencias, todos los programas tienen una jornada en donde los practicantes presentan a sus compañeros y asesores sus estudios o intervenciones al final del semestre. Solo en un programa se evidenció motivación a los estudiantes a presentar sus estudios en eventos de corte nacional.

\section{Conclusiones y recomendaciones}

La realización de este estudio descriptivo permitió identificar, en términos generales, las siguientes fortalezas en la formación investigativa de los practicantes. Por un lado, se destaca la conceptualización de la práctica pedagógica dentro del paradigma de la acción investigativa, desde el Reglamento de Práctica de la Facultad de Educación. Sin duda, esto representa un avance frente a la tradición de considerar la práctica tan solo como la oportunidad para aplicar los conocimientos teóricos que el practicante recibe en los cursos de pedagogía y didáctica. Se necesita empoderar al futuro docente con las competencias necesarias que le permitan adentrarse en el conocimiento crítico de su realidad educativa, buscando el mejoramiento permanente de los procesos de enseñanza y aprendizaje a partir del desarrollo de proyectos investigativos. Autores como Chacón (2006), Domínguez y Raposo (citados por Pérez, Benarroch, Jiménez, Smith y Rojas, 2006). (2006), entre otros, ratifican la importancia de privilegiar en el ejercicio de la práctica pedagógica el desarrollo de una cultura investigativa, que le permita al docente reflexionar y transformar su realidad a partir de la solución de problemáticas que identifique en su contexto educativo.

En el mismo sentido, tanto los coordinadores como los asesores reconocen la importancia de la investigación en el contexto de la práctica pedagógica. Señalan que este tipo de experiencia no solo les permite a los practicantes tomar conciencia del rol que deben asumir como docentes autocríticos, sino también buscar soluciones prácticas a las situaciones problemáticas que deben enfrentar en su quehacer docente. No obstante, algunos practicantes se mues- tran particularmente críticos sobre las falencias que se presentan en la articulación de la práctica y la investigación porque sus iniciativas responden más a sus intereses personales sobre determinados temas que a una necesidad real de su contexto educativo. Esto a su vez los lleva a cuestionar el bajo impacto de sus experiencias en la comunidad educativa en donde desarrollan su práctica. Dicha percepción está sustentada en el documento expedido por el MEN (2012), Políticas y sistema colombiano de formación $y$ desarrollo profesional docente, en donde se establece que hay una desarticulación entre la investigación y la práctica pedagógica en los procesos de formación docente, y además se señala que los proyectos investigativos son más de carácter disciplinar, y no se enfocan en los contextos y las dinámicas cotidianas de la escuela.

En el terreno de la praxis, se evidencia la preocupación de la mayoría de los programas académicos por articular la experiencia de la práctica pedagógica con los procesos investigativos a través de proyectos de investigación acción en el aula, estudios de caso, estudios descriptivos, entre otros. Sin embargo, no en todos los programas esta articulación se cumple plenamente, a pesar de que en los planes de estudio se han incorporado cursos como Epistemología, Metodología de la Investigación, Seminario de Investigación y se promuevela conformación de grupos y semilleros de investigación. Al respecto, uno de los coordinadores sugiere que debería ser un requisito de grado la formulación de un proyecto de investigación para que se desarrolle en la práctica pedagógica, debido a que desafortunadamente no todos los estudiantes toman esta experiencia con la seriedad que requiere.

Por otro lado, se advierte en la mayoría proyectos analizados la falta de un desarrollo balanceado de todas las competencias investigativas. En algunos casos, se registra la ejecución de las competencias observacionales por parte de los practicantes, pero no se evidencia la sistematización de los datos que se recogen a partir de esta experiencia en algún formato especial. También se identifican dificultades en la formulación de la pregunta investigativa ya que 
no se plantea un cuestionamiento claro que se pueda responder a partir de los proyectos propuestos. En cuanto a la competencia procedimental, no todos los reportes incluyen los pasos básicos que deben orientar este proceso; por ejemplo, en algunos casos no se establece con claridad el diseño metodológico que se siguió en el proyecto. Tampoco se encuentra en la mayoría de los reportes analizados el uso de herramientas tecnológicas para realizar el análisis de los resultados de los estudios. Finalmente, se registran falencias en las competencias escriturales de los practicantes a nivel de la redacción de los informes investigativos e inconsistencias en su estructuración. Este panorama nos lleva a reflexionar en que, a pesar del esfuerzo que están haciendo los programas de la facultad, no se logra concretar la propuesta de Bartlett (1994), que incluye los componentes esenciales del proceso investigativo que los docentes en formación pueden desarrollar en el contexto de la práctica pedagógica: observación y recolección de evidencias, en donde se pone en práctica el uso de diferentes instrumentos; interpretación de la información desde una perspectiva crítica sobre el quehacer docente; identificación de aspectos relevantes que ameriten intervención; búsqueda de alternativas de acción a partir de una nueva forma de entender la enseñanza, para finalizar el proceso con la implementación de nuevas estrategias, cuyo propósito fundamental es el enriquecimiento del proceso de aprendizaje de los alumnos, y así contribuir con el mejoramiento de la calidad de la educación en nuestro país.

Recogiendo la percepción de los propios actores de la práctica pedagógica, ellos consideran que el Comité de la Facultad, los coordinadores de los programas, asesores, cooperadores y estudiantes deberían trabajar mancomunadamente para alcanzar los objetivos propuestos. Esto se podría lograr a través del aprovechamiento de espacios como la semana de inducción y las mismas asesorías para promover experiencias más significativas orientadas a la reflexión, autocrítica y solución de problemas puntuales y relevantes, que puedan tener mayor impacto en la comunidad académica en donde los estudiantes desarrollan su práctica. La apertura de estos espacios y el trabajo colaborativo entre los participantes contribuiría no solo a un mejor desarrollo de las competencias investigativas interpersonales de los practicantes -que según Aular de Durán, Marcano y Moronta (2009) implica la capacidad para relacionarse armónicamente entre los sujetos implicados dentro del proceso-, sino que también mejoraría la calidad de los productos logrados durante la práctica docente. A esto se suman Ollarves y Salguero (2009), quienes afirman que todas las actividades y decisiones tomadas necesitan la aprobación, inclusión, participación y coordinación de los miembros involucrados para que los productos, eventos o servicios generados sean más confiables, ya que han sido sometidos a diversos juicios.

Por otra parte, se requiere espacios de desarrollo profesional docente que involucren a los asesores en la actualización de sus conocimientos de los procesos investigativos, y particularmente con los procesos de retroalimentación, de tal manera que todos podamos hablar el mismo lenguaje. El MEN (2012) confirmó que, según los estudios realizados, la formación investigativa de los docentes en ejercicio en nuestro país suele ser débil, poco sistemática y fragmentada. No obstante, el MEN se encuentra trabajando en políticas de desarrollo profesional para los docentes, y trabajos como el presente estudio corroboran la necesidad de fortalecer desde las universidades y en especial desde las licenciaturas, la capacitación de docentes vinculados con el sector educativo y en particular aquellos que se desempeñan como formadores de docentes en etapa inicial.

Hace falta crear más conciencia en los estudiantes acerca de que la práctica se debe asumir como un espacio de crecimiento y aprendizaje que consiste, no únicamente en transmitir el conocimiento científico adquirido en el campo específico, sino también en poner en práctica todas las herramientas y estrategias aprendidas en áreas como la investigativa, que le permitan hacer de esta experiencia un espacio enriquecedor para el beneficio de sus aprendices y de la comunidad educativa. Esto acentúa la urgencia de establecer objetivos claros para la práctica docente y lograrlos en su totalidad, puesto que según Cid, Domínguez 
y Raposo (citados por Pérez, Benarroch, Jiménez Smith y Rojas, 2006) uno de los objetivos más importantes de la práctica es permitir al estudiante adquirir hábitos de investigación que lo impulsen a construir su conocimiento profesional y así, a partir de la valoración crítica de sus vivencias, identificar problemáticas y tomar decisiones para resolverlas.

Amerita la revisión de los contenidos y las prácticas de los cursos que están orientados a empoderar a los estudiantes como docentes reflexivos, con un espíritu investigativo, debido a que se evidencian algunos vacíos que no permiten cumplir plenamente las expectativas de la Facultad de Educación. En algunos casos los docentes practicantes no diferencian entre un proyecto pedagógico y un proyecto investigativo, no todos tienen claridad en la conceptualización de todos los elementos o etapas que conlleva el proceso investigativo. Algunos confunden diseño metodológico de la investigación con los principios metodológicos de enseñanza. Muñoz, Quintero y Munévar (2001) afirman que aunque la investigación representa un reto para la educación del siglo XxI, es el camino viable para generar conocimientos, mejorar la labor docente y por ende el proceso de enseñanza-aprendizaje en las instituciones educativas. Esto hace prioritario continuar la búsqueda de estrategias que permitan una mejor articulación de la práctica y los cursos de investigación orientados en las licenciaturas.

Por otro lado, es deseable que desde la Facultad de Educación se garantice la continuidad en la edición de la revista Reflexiones Pedagógicas para que a través de este medio se visibilicen los resultados de las experiencias significativas de los practicantes y se pueda tener un mayor impacto en la comunidad académica. Esto influiría positivamente en el desarrollo de las competencias investigativas comunicativas que según Balvo (2010) demuestran la habilidad del investigador para generar y difundir conocimientos a partir de su investigación, que posteriormente contribuirán al intercambio de experiencias y por ende al enriquecimiento mutuo entre los miembros de una determinada comunidad educativa (Ollarves y Salguero, 2009).
Finalmente, con la realización de este trabajo investigativo se fijan las bases para el diseño, la implementación y el seguimiento de una subsiguiente propuesta de intervención que asuma como punto de partida la reconceptualización de la práctica pedagógica como un espacio formativo que les permita a los practicantes entrar en interacción con las instituciones educativas y buscar desde allí la transformación de los procesos educativos a través de la reflexión y la investigación (Chacón, 2006), yendo más allá del sentido convencional de considerar a la práctica pedagógica tan solo como una oportunidad para aplicar los conocimientos pedagógicos, didácticos y disciplinares adquiridos durante el periodo de formación inicial.

\section{Referencias}

Ary, D., Jacobs, L. y Sorensen, C. (2010). Introduction to research in education. Wadsworth: Cengage Learning.

Aular de Durán, J., Marcano, N. y Moronta, M. (2009). Competencias investigativas del docente de educación básica. Laurus, 15 (30), 138-165.

Balvo, J. (2010). Formación en competencias investigativas, un nuevo reto de las universidades. Universidad Nacional Experimental del Táchira.

Bartlett, L. (1994). Teacher development through reflective teaching. En J. Richards y D. Nunan (ed.), Second Language Teacher Education. Nueva York: Cambridge University Press.

Bowen, G. (2009). Document analysis as a qualitative research method. Qualitative Research Journal, 9(2), pp. 27-40. Recuperado de http://www.academia. edu/8434566/Document_Analysis_as_a_Qualitative_ Research_Method

Calvo, G. (2002). El docente: responsable de la investigación pedagógica. Recuperado de http://www. banrepcultural.org/blaavirtual/educacion/expedocen/ expedocen $5 \mathrm{c} . \mathrm{htm}$

Castillo, S. (2011). Evaluación de competencias investigativas. XIII Conferencia Interamericana de Educación Matemática. Recife.

Chacón, M. A. (2006). La enseñanza reflexiva en la formación de los estudiantes de pasantías de la carrera de Educación Básica Integral. Recuperado de http://www. tesisenxarxa.net/TDX-0625107- 120634/index.html 
Consejo de Facultad de Educación de la Universidad Surcolombiana. (2013). Acuerdo Número 072 de 2013. Reglamento de Práctica de la Facultad de Educación, p. 3.

Corcoran, C. y Leahy, R. (2003). Growing professionally through reflective practice. Recuperado de http://files. eric.ed.gov/fulltext/EJ787758.pdf

Dewey, J. (1909). How we think. Boston: D. C. Heath.

Edukanda. (s. f.). Cómo mejorar mis competencias profesionales. Recuperado de www.edukanda.es/ mediatecaweb/data/zip/1261/page_11.htm

Farrell, T. (2003). Reflective teaching: The principles and practices. English Teaching Forum, 41 (4), 14-21.

García, M. y Veleros, M. del C. (2012). Competencias reflexivas y didácticas en profesores universitarios: Construcción de un portafolio electrónico. Tendencias Pedagógicas, 20.

Hernández, R., Fernández, C. y Baptista, P. L. (2006). Metodología de la investigación. México: McGraw-Hill.

Insuasty, E. A. y Zambrano, L. C. (2010). Exploring reflective teaching through informed journal keeping and blog group discussion in the teaching practicum. Profile, 12 (2), pp. 87-105.

Insuasty, E. A. y Zambrano, L. C. (2011). Caracterización de los procesos de retroalimentación en la práctica docente. Entornos, 24, 73-85.

Jansen, H. (2010). The logic of qualitative survey research and its position in the field of social research methods. Qualitative Social Research Journal, 11(2). Recuperado de http://www.qualitative-research.net/index.php/fqs/ article/view/1450/2946

Jurado, F. (2003). ¿Son las competencias el nuevo enfoque que la educación requiere? Magisterio, 1, 14-16.

Kvale, S. (2008). Interviews: Learning the craft of qualitative interviewing. Thousand Oaks: Sage.

Luque, D., Quintero, C. A. y Villalobos, F. (2012). Desarrollo de competencias investigativas básicas mediante el aprendizaje basado en proyectos como estrategia de enseñanza. Actualidades Pedagógicas, $60,29-49$.

Mercado, E. (2003). Del estudiante a maestro practicante: los ritos en las prácticas pedagógicas en la Escuela Normal. Tiempo de Educar, 4 (7).
Ministerio de Educación Nacional. (2012). Políticas y sistema colombiano de formación y desarrollo profesional docente. Bogotá. Recuperado de http://www. colombiaaprende.edu.co/html/productos/1685/ articles-312233_documentobaseop.pdf

Ministerio de Educación Nacional. (2014). Lineamientos de calidad para las licenciaturas en educación. Bogotá. Recuperado de https://www.mineducacion.gov. co/1759/articles-357233_recurso_1.pdf

Montoya, J. (2006). Origen, concepto y tipos de competencias. Recuperado de http://es.slideshare.net/joma72/ origen-concepto-y-tipos-de-competencias

Muñoz, J., Quintero, J. y Munevar, R. (2001). Cómo desarrollar competencias investigativas en educación. Bogotá: Cooperativa Editorial Magisterio.

Ollarves, Y. y Salguero, L. (2009). Una propuesta de competencias investigativas para los docentes universitarios. Laurus, 15 (30), 33-50.

Pérez, M., Benarroch, A., Jiménez, M. A., Smith, G. y Rojas, G. (2006). ¿Se puede estimular la reflexión en el supervisor y en el alumno universitario durante el periodo de Prácticum? Enseñanza, 24, 33-51.

Porta, L. y Silva, M. (2003). La investigación cualitativa: el análisis de contenido en la investigación educativa. Recuperado de http://www.uccor.edu.ar/paginas/ REDUC/porta.pdf

Restrepo, B. (s. f.). Una variante pedagógica de la investigación-acción educativa. Recuperado de http:// cit.uao.edu.co/docente/sites/default/files/repositorio/Investigaci $\% \mathrm{C} 3 \% \mathrm{~B} 3 \mathrm{n} \% 20$ acci\%C3\%B3n\%20 Pedag\%C3\%B3gica\%20-\%20B\%20Restrepo.pdf

Rodrigues, B., Hoffmann M. y Mackedanz F. (2011). Cómo investigar cualitativamente. Entrevista y cuestionario. Contribuciones a las Ciencias Sociales. Recuperado de www.eumed.net/rev/cccss/11/

Seliger, H. W. y Shohammy, E. (1989). Second language research method. Oxford: Oxford University Press.

Villarini, Á. (2014). Un modelo del pensamiento reflexivo, creativo y crítico como competencia humana general. Revista Internacional Magisterio, 66.

Zambrano, L. C. e Insuasty, E. A. (2008). Analysis of the teaching practicum in the light of the reflective teaching approach. Lenguaje, 36 (2), 447-471.

Para citar este artículo

Buendía-Arias, X. P., Zambrano-Castillo, L. C, y Insuasty, E. A. (2018). El desarrollo de competencias investigativas de los docentes en formación en el contexto de la práctica pedagógica. Folios, 47, 179-195. 